\title{
Validity of Human Milk as regards Arakawa's Reaction.
}

\author{
52nd Report of the Peroxidase Reaction.
}

$\mathrm{By}$

Yoshitaka Uga.

(宇贺義孝)

(From the Department of Pediatrics, Faculty of Medicine, Tohoku

Imperial University, Sendai. Director: Prof. A. Sato.)

It is a matter of course that raw human milk will, if left to itself, soon undergo a number of changes, chemical as well as physical. I wanted to test with Araka wa's reagents the change of raw human milk with respect to lapse of time.

The result of such an experiment will contribute to the practice of Arakawa's's2) reaction, because we cannot, though it is desirable to test as soon as possible the Araka w a reaction of human milk in a clinical case, always perform the test as soon as we have obtained a sample of milk from a breast. It can be seen by common sense that a sample of milk, obtained to-day and tested soon enough, will be different in intensity of the A rak a w a reaction from the same sample tested to-morrow. The result of the present trial will thus show how long a sample of raw human milk may be left to itself without a change, as far as Arakawa's reaction is concerned.

\section{Method.}

Material. Milk from one and the same lactant with a rather abundant secretion and a healthy baby was always used, because it was neeessary for this experiment to use a rather large amount of milk. Breasts were cleaned with sufficient care, and almost 10 c.c. of milk obtained from each breast. The time of obtaining was about 8 o'clock in the morning.

1) T. A rak aw a, Tohoku J. Exp. Med., 1930, 16, 83 and 107.

2) A. S a to, Jikkeniho, 1933, 228, 1554. 
Preservation. Milk samples thus obtained were either left at the room temperature or kept in an ice box, except during the time of performance of the Arakaw a reaction, which was of course of a short duration.

\section{Experiment I. Preliminary Experiment.}

Such material as is left to itself at room temperature must, as will be easily imagined, change very soon in summer. So I tried the Arakawa reaction of a portion out of one and the same sample of human milk, immediately after the sample was obtained from a breast. The sample was then left at room temperature and the same reaction was tried one, two and three hours later. The result is shown in Table I (Cf. Table I).

\section{TABLE I.}

Change of the sane samples of the human milk from a lactant left at room temperature in summer, as shown by means of Ar alk aw a's reaction.

\begin{tabular}{|c|c|c|c|c|}
\hline \multirow[b]{2}{*}{ Date } & \multirow[b]{2}{*}{ Time } & \multirow{2}{*}{$\begin{array}{l}\text { Room } \\
\text { tempera- } \\
\text { ture }\end{array}$} & \multicolumn{2}{|c|}{ Arakawa's reaction } \\
\hline & & & $\begin{array}{c}\text { Right } \\
\left(0^{\prime}\right)\left(1^{\prime}\right)\left(2^{\prime}\right)\left(3^{\prime}\right)\left(4^{\prime}\right)\left(5^{\prime}\right)\end{array}$ & $\begin{array}{c}\text { Left } \\
\left(0^{\prime}\right)\left(1^{\prime}\right)\left(2^{\prime}\right)\left(3^{\prime}\right)\left(4^{\prime}\right)\left(5^{\prime}\right)\end{array}$ \\
\hline \multirow{4}{*}{$\begin{array}{l}8 . \\
\mathrm{IX}\end{array}$} & 8A.M. & $23^{\circ} \mathrm{C}$ & $(t)(H H)(H t)(H H)(H+)(H t)$ & $( \pm)(t+)(t+)(H+)(H+)(H)$ \\
\hline & 9 A.M & & (H) (H) (H) (Ht) (H) & $( \pm)(t+)(+t)(H)(H+)(H)$ \\
\hline & 10 A.M. & $23^{\circ} \mathrm{C}$ & $(+)(H)$ (H) (HH) (H) (Ht) & $( \pm)(++)(++)(H)(H)(H)$ \\
\hline & $\begin{array}{l}11 \text { A.M. } \\
8 \text { A.M. }\end{array}$ & $\begin{array}{l}27^{\circ} \mathrm{C} \\
23^{\circ} \mathrm{C}\end{array}$ & $(+)(H)(H)(H+)(H)(H+)$ & $\begin{array}{l}( \pm)(+t)(+t)(H)(H)(H) \\
(+)(+t)(+t)(H+)(H+)(H)\end{array}$ \\
\hline \multirow{4}{*}{$\begin{array}{c}6 . \\
I X\end{array}$} & 9 A.M. & $23^{\circ} \mathrm{C}$ & $(+)(+t)(H)(H)(H+)(H)$ & $(+)(+t)(H+)(H+)(H)(H+)$ \\
\hline & 10 A.M. & $24^{\circ} \mathrm{C}$ & $(+)(+t)(H)(H)(H)(H)$ & $(+)(+t)(\mathrm{HH})(\mathrm{HH})(\mathrm{Ht})(\mathrm{HH})$ \\
\hline & 11 A.M. & $25^{\circ} \mathrm{C}$ & $(+)(+t)(H+)(H)(H+(H)$ & $(+)(t+)(H)(H+)(H+)(H)$ \\
\hline & 8 & $25^{\circ} \mathrm{C}$ & $(+)(t+)(H+)(H)(H)(H)$ & $( \pm)(+)(+t)(+t)(+1)(H)$ \\
\hline 26. & 9 A.M. & $26^{\circ} \mathrm{C}$ & $(+)(t+)(H)(H+)(H t)(H+)$ & $( \pm)(t)(t+)(t+)(H t)(H t)$ \\
\hline \multirow[t]{3}{*}{ VIII } & 10 A.M. & $27^{\circ} \mathrm{C}$ & $(+)(\mathrm{H})(\mathrm{HH})(\mathrm{HH})(\mathrm{HI})(\mathrm{HH})$ & $( \pm)(+)(t+)(t+)(H)(H)$ \\
\hline & 11 A.M. & $27^{\circ} \mathrm{C}$ & $(+)(t+)(H)(H)(H)(H)$ & $( \pm)(t)(t+)(t+)(H)(H+)$ \\
\hline & & $20^{\circ} \mathrm{C}$ & $(+)(+t)(\mathrm{HH})(\mathrm{HH})(\mathrm{HH})(\mathrm{HH})$ & $(+)(+t)(H+1)(H)(H+)(H+)$ \\
\hline \multirow{4}{*}{$\begin{array}{c}4 . \\
\text { vII }\end{array}$} & 9 A.M. & $22^{\circ} \mathrm{C}$ & $(+)(t+)(H+)(H+)(H)(H)$ & $(+)(t+)(H)(H)(H)(H)$ \\
\hline & 10 A.M. & $22^{\circ} \mathrm{C}$ & $(+)(+t)(H)(H+)(H)(H)$ & $(+)(+t)(H+1)(H)(+H)(H+)$ \\
\hline & $11 \mathrm{~A} . \mathrm{I}$ & & $(+)(+t)(H)(H+)(H)(H)$ & $(+)(+t)(+H)(+t+)(+H)(+H)$ \\
\hline & & $23^{\circ} \mathrm{C}$ & $(t)(+)(t+)(t+)(t+)(t+)$ & $(+)(+t)(H+H)(H+)(H+)(H)$ \\
\hline \multirow{3}{*}{$\begin{array}{l}15 . \\
\text { VII }\end{array}$} & 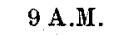 & $24^{\circ} \mathrm{C}$ & $( \pm)(+)(t+)(t+)(t+)(t+)$ & $(+)(+t)(H)(H)(H+(H)$ \\
\hline & 10 A.M. & $25^{\circ} \mathrm{C}$ & $( \pm)(t)(t+)(t+)(t+)(t+)$ & $(+)(+t)(+H)(+t+)(+H)(+H)$ \\
\hline & 11 A.M. & $25^{\circ} \mathrm{C}$ & $(t)(t t)(t+)(t+)(t t)$ & $(+)(+t)(+H)(H+)(+H)(H)$ \\
\hline
\end{tabular}

The result shown in Table $I$ is rather striking, because human milk left to itself at room temperature in summer time did not, as far as Arakawa's reaction was concerned, show a change as long as 3 hours, which was rather against our expectation. 
This result shows that human milk may be tested with Ara$\mathrm{k}$ awa's reaction with the same result even as late as three hours after obtaining even in summer time.

\section{Experiment $I I$.}

In the experiment proper one and the same samples of human milk were tested 4 times : namely, 8 A.M., immediately after obtaining, 11 A.M. ( 3 hours later), 1 P.M. (5 hours later), and 4 P.M. (8 hours later).

\section{1st Series of Experiment.}

Milk samples left at room temperature. The results will be seen from Table II (Cf. Table II).

\section{TABLE II.}

Change of the same samples of the human milk from a lactant at room temperature, as shown by means of Araka wa's reaction.

\begin{tabular}{|c|c|c|c|c|}
\hline \multirow{2}{*}{ Date } & \multirow{2}{*}{ Time } & \multirow{2}{*}{$\begin{array}{c}\text { Room } \\
\text { tempera- } \\
\text { ture }\end{array}$} & \multicolumn{2}{|c|}{ Arakawa's reaction } \\
\hline & & & $\begin{array}{c}\text { Right } \\
\left(0^{\prime}\right)\left(1^{\prime}\right)\left(2^{\prime}\right)\left(3^{\prime}\right)\left(4^{\prime}\right)\left(5^{\prime}\right)\end{array}$ & $\begin{array}{c}\text { Left } \\
\left(0^{\prime}\right)\left(1^{\prime}\right)\left(2^{\prime}\right)\left(3^{\prime}\right)\left(4^{\prime}\right)\left(5^{\prime}\right)\end{array}$ \\
\hline & & & $(+)(+t)(+H)(H t)(H+H)(H)$ & $( \pm)(t+)(H+)(H)(t+$ \\
\hline 8. & & & (HI) (HI) (HI) (HH) & $( \pm)(+t)(H+)$ \\
\hline IX & & & $(+)(+t)(+H)(+H)(H t)(+H)$ & $( \pm)(+)(+t)(+t)(+H)(H)$ \\
\hline & & & $\begin{array}{l}(+)(+t)(H+)(H+)(H)(H) \\
(+)(H)(H+)(H)\end{array}$ & $( \pm)(+)(+t)(+t)(+t)(+t)$ \\
\hline б. & 112 & $c$ & 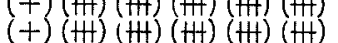 & $\begin{array}{l}(+)(H)(H)(H+)(H+)(H+) \\
(+)(H+)(H+)(H+)(H+)(H+)\end{array}$ \\
\hline VIII & & & $(+)(H)(H)(W)(H+)(H)$ & $(+)(t+)(+t)(H+)(H+)(H+)$ \\
\hline & & & $\left\{\begin{array}{l}(+)(+t)(H)(H)(H)(H) \\
+)(+t)(H)(H)(H)(H)\end{array}\right.$ & $\begin{array}{l}( \pm)(+)(+t)(+t)(+t)(+H) \\
( \pm)(+)(t+)(+t)(+t)(+4)\end{array}$ \\
\hline 96 & $11 \mathrm{~A}$ & ${ }^{\circ} \mathrm{C}$ & 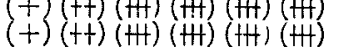 & 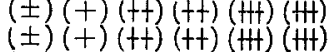 \\
\hline VIII & & & $(+)(+t)(H+)(H)(H)(H)$ & $( \pm)(+)(t+)(+t)(+t)(+t)$ \\
\hline & & & $(+)(+t)(H)(H)(H)(H)$ & $( \pm)(+)(++)(++)(++)(+)$ \\
\hline 6. & $\begin{aligned} 8 \mathrm{~A} \\
11 \mathrm{~A}\end{aligned}$ & $\mathrm{C}$ & 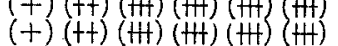 & 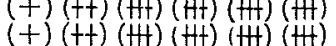 \\
\hline IX & & & $(+)(++)(H+)(H+)(H)(H)$ & $(+)(+t)(H t(H)(H)(H t)$ \\
\hline & & & $(+)(+t)(H)(H)(H)(H)$ & $(+)(t+)(+H)(+H)(+t)(+H)$ \\
\hline 4. & $11 \mathrm{~A}$ & $17^{\circ} \mathrm{C}$ & $( \pm)(+t)(H)(H)(H)(H)$ & $( \pm)(+)(t+)(H t)(H)(H)$ \\
\hline $\mathbf{X}$ & & & $( \pm)(+)(+t)(+W)(+W)(+\#)$ & $(-)(+)(++)(H+)(+H)(+H)$ \\
\hline & & & 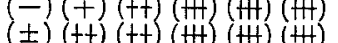 & 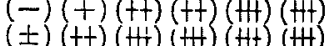 \\
\hline $\mathbf{5}$ & $11 \mathrm{~A}$ & & $( \pm 3(+t)(+t)(H+)(H)(H)$ & $( \pm)(+t)(H+)(H)(H+H)(+\#)$ \\
\hline & & & $(-)(+)(+t)(t+)(t+)(+t)$ & $( \pm\{(+t)(H+\}(+H)(+W)(W)$ \\
\hline & & & $(-)(+)(t+)(+t)(+t)(+W)$ & $( \pm)(+t)(+t)(H+)(+H)(+H)$ \\
\hline & & & (开) & $( \pm)(+t)(H+)(H)(H)(H+)$ \\
\hline $\mathrm{x}$ & & & $( \pm)(t+)(+H)(+H)(H)(H)$ & $(-\{(+)(t+)(t+)(t+)(t+)$ \\
\hline & & & $( \pm)(t+)(H+)(H)(H)(H)$ & $(-)(t)(t+)(t+)(t+$ \\
\hline
\end{tabular}


Validity of Human Milk as regards A rak a wa's Reaction

\begin{tabular}{|c|c|c|c|c|}
\hline \multirow{2}{*}{ Date } & \multirow{2}{*}{ ime } & \multirow{2}{*}{$\begin{array}{c}\text { Room } \\
\text { tempera- } \\
\text { tur' }\end{array}$} & \multicolumn{2}{|c|}{ Arakawa's reaction } \\
\hline & & & $\begin{array}{c}\text { Right } \\
\left(0^{\prime}\right)\left(1^{\prime}\right)\left(2^{\prime}\right)\left(3^{\prime}\right)\left(4^{\prime}\right)\left(5^{\prime}\right)\end{array}$ & $\begin{array}{l}\text { Left } \\
\left(0^{\prime} \quad\left(1^{\prime}\right)\left(2^{\prime}\right)\left(3^{\prime}\right)\left(4^{\prime}\right)\left(5^{\prime}\right)\right.\end{array}$ \\
\hline & $8 \mathrm{~A}, \mathrm{I}$ & $18^{\circ} \mathrm{C}$ & $(\mathrm{HH})(\mathrm{H})(\mathrm{H})$ & $( \pm)(t+)(H)(H t)(H t)(H)$ \\
\hline 11. & 11 A.M. & $20^{\circ} \mathrm{C}$ & $H+(H)(H)(H)$ & tt) (Ht) (Ht)(Ht) (Ht) \\
\hline $\mathbf{X}$ & 1 P.MI. & $21^{\circ} \mathrm{C}$ & $(t+)(t+)(H)(H)$ & $( \pm)(t+)(H t)(H t)(H t)(H)$ \\
\hline & $\begin{array}{l}4 \text { P.M. } \\
8 \text { A.M. }\end{array}$ & $\begin{array}{l}20^{\circ} \mathrm{C} \\
17^{\circ} \mathrm{C}\end{array}$ & $(+t)(+t)(t+)(t+)$ & $( \pm)(+t)(H H)(H t)(H)(H)$ \\
\hline 18. & 11 A.M. & $19^{\circ} \mathrm{C}$ & 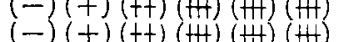 & $\begin{array}{l}( \pm)(t t)(t+)(H)(H t)(H \\
( \pm)(t t)(H t)(H t)(H t)(H\end{array}$ \\
\hline $\mathbf{X}$ & 1 P.M. & $20^{\circ} \mathrm{C}$ & $+)(+t)(+t)(H)(H)$ & $( \pm)(t+)(t+)(t+)(H t)(H)$ \\
\hline & 4 P.M. & $20^{\circ} \mathrm{C}$ & $+)(+t)(+t)(+H)(H)$ & $( \pm)(t+)(t+)(t+)(+H)(H)$ \\
\hline 6. & $\begin{array}{l}8 \text { A.M. } \\
11 \text { A.M. }\end{array}$ & $\begin{array}{l}19^{\circ} \mathrm{C} \\
19^{\circ} \mathrm{C}\end{array}$ & $\begin{array}{l}t)(H+)(H)(H)(H+) \\
t)(H)(H)(H)(H)\end{array}$ & $(-)(+)(++)(H)(H)(H)$ \\
\hline XII & 1 P.M. & $19^{\circ} \mathrm{C}$ & t) (H) (H) (H) (H) & $(-)(+)(t+)(H t)(H)(H)$ \\
\hline & 4 P.M. & $20^{\circ} \mathrm{C}$ & t+) (HH) (HH) (H) (HH) & $(-)(+)(t+)(H t)(t H)(t+)$ \\
\hline & 8 A.M. & $14^{\circ} \mathrm{C}$ & $\pm)(+t)(H)(H)(H)$ & $(-)(t+)(t+)(H t)(H t)(H+)$ \\
\hline 9. & 11 A.M. & $14^{\circ} \mathrm{C}$ & t) (tt) (HH) (H) (H) & $(-)(+t)(t+)(H+)(H+)(H t)$ \\
\hline XII & 1 P.M. & $15^{\circ} \mathrm{C}$ & $t)(t+)(H)(H+)(H)$ & $(-)(t+)(t+)(H t)(H+)(H)$ \\
\hline & 4 P.M. & $19^{\circ} \mathrm{C}$ & $+)(t+)(t+)(+H)(H)$ & $(-)(+t)(+t)(+H)(H)(H)$ \\
\hline & 8 A.M. & $13^{\circ} \mathrm{C}$ & $\pm(+)(+t)(H+1)(H)$ & $(-)(t+)(t+)(H t)(H)(H)$ \\
\hline 15. & 11 A.M. & $14^{\circ} \mathrm{C}$ & $\pm(+)(+t)(H)(H)$ & $(-)(+t)(+t)(H+)(H)(H)$ \\
\hline XII & 1 P.M. & $15^{\circ} \mathrm{C}$ & $\begin{array}{l}t)(t+)(t+)(t+)(H+) \\
t)(t+)(t+)(H+(H)\end{array}$ & $(-)(+t)(+t)(H+)(H)(H)$ \\
\hline & $\begin{array}{l}4 \text { P.M. } \\
\text { S A.M. }\end{array}$ & $\begin{array}{l}19^{\circ} \mathrm{C} \\
19^{\circ} \mathrm{C}\end{array}$ & $\begin{array}{l}(t+)(+t)(H)(H) \\
(H+)(H)(H)(H)\end{array}$ & 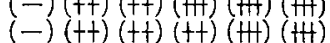 \\
\hline 17. & 11 A.M. & $20^{\circ} \mathrm{C}$ & $( \pm)(t+)(H t)(H+)(H)(H)$ & $(-)(t+)(t+)(t+)(+H)(+\#)$ \\
\hline XII & 1 P.M. & $20^{\circ} \mathrm{C}$ & $( \pm)(+t)(H)(H)(H)(H)$ & $(-)(t+)(t+)(t+)(H t)(H)$ \\
\hline & 4 P.M. & $23^{\circ} \mathrm{C}$ & $(+t)(H+)(H)(H)(H)$ & $(-)(t+)(+t)(t+)(+H)(+t)$ \\
\hline & 8A.M. & $20^{\circ} \mathrm{C}$ & (H) (H) (H) (H) & $( \pm)(t+)(H)(H)(H)(H+)$ \\
\hline 19. & 11 A.M. & $21^{\circ} \mathrm{C}$ & (H) (H) (H) (H) & $( \pm)(t+)(H+(H t)(H)(H)$ \\
\hline XII & 1 P.M. & $27^{\circ} \mathrm{C}$ & $(+t)(H+)(H)(H)(H)$ & $( \pm)(+t)(H)(H t)(H)(H)$ \\
\hline & 4 P.M. & $24^{\circ} \mathrm{C}$ & $(t+)(H)(H)(H)(H)$ & $( \pm)(t+)(H+)(H)(H)(H)$ \\
\hline & 8A.M. & $16^{\circ} \mathrm{C}$ & $(t+)(t+)(H)(H)$ & $(-)(+)(t+)(t+)(+4)(+H)$ \\
\hline 17. & 11 A.M. & $17^{\circ} \mathrm{C}$ & $(t+)(t+)(H+)(t+t)$ & $(\mathrm{tH})(\mathrm{H})$ \\
\hline I & $\mathrm{M}$. & $18^{\circ} \mathrm{C}$ & $t+)(+t)(H+)(H)$ & $(t+)(t+)$ \\
\hline & & 17 & tt) $(t+)(t+)(H+)$ & is \\
\hline & 8 A.M. & 17 & (Hit) (H+) (H) & $\ddot{3}$ \\
\hline 20. & 11 A.M. & $19^{\circ} \mathrm{C}$ & $+t)(H)(H)(H)$ & $(t+)(t+)$ \\
\hline $\mathbf{T}$ & M. & $19^{\circ} \mathrm{C}$ & $(+t)(H)(H)(H)$ & $(t+)(t+)$ \\
\hline & 41 & & $(+t)(\mathrm{H})(\mathrm{Ht})(\mathrm{HH})$ & $(++)$ \\
\hline 27. & $\begin{array}{l}\text { 8 A.M. } \\
11 \text { A.M. }\end{array}$ & $\begin{array}{l}17^{\circ} \mathrm{C} \\
19^{\circ} \mathrm{C}\end{array}$ & $\begin{array}{l}+t)(\mathrm{HH})(\mathrm{HH})(\mathrm{Htt}) \\
(t)(\mathrm{HH})(\mathrm{Ht})(\mathrm{Ht})\end{array}$ & $\begin{array}{l}-)(t+)(+H)(+H)(H) \\
-)(t+)(H)(H)(H)\end{array}$ \\
\hline $\mathbf{I}$ & 1 P.M. & $20^{\circ} \mathrm{C}$ & $(t+)(t+)(H+)(H)$ & $+f(+t)(+t)(+H)(H)$ \\
\hline & 41 & $19^{\circ} \mathrm{C}$ & $(+)(t+)(H)(H)$ & $+)(t+)(+t)(H)(H)$ \\
\hline & 8 & & $(\mathrm{tt})(\mathrm{HH})(\mathrm{HH})$ & $(\mathrm{H})(\mathrm{Ht})(\mathrm{H})(\mathrm{HH})(\mathrm{H})$ \\
\hline 18. & 11 A.M. & $21^{\circ} \mathrm{C}$ & $(t+)(t+)(H)(H)$ & $(+t)(H)(H)(H t)(H)$ \\
\hline $\mathbf{I}$ & 1 P.M. & $22^{\circ} \mathrm{C}$ & $t+)(t+)(t+)(H)$ & $(+t)(H)(H)(H)(H)$ \\
\hline & 41 & $24^{\circ} \mathrm{C}$ & $(t+)(t+)(H)$ & $(+t)(H+)(H)(H)(H)$ \\
\hline & 8 & & H) (HH) (H) (H) & $\int(t+)(t t)(t+)(t t$ \\
\hline 22. & 11 A.M. & $19^{\circ} \mathrm{C}$ & f) $(\mathrm{tH})(\mathrm{HH})(\mathrm{H})$ & $t)(t+)(t t)(t+)(t+)$ \\
\hline I & 1 P.M. & $19^{\circ} \mathrm{C}$ & Hit) $(H+1+1+$ & $(t)(t+)(t+)$ \\
\hline & 4 P.M. & $19^{\circ} \mathrm{C}$ & $H+)(H)(H)$ & $+5(+)(+)(+t)$ \\
\hline & 8 A.MI. & $17^{\circ} \mathrm{C}$ & $(t+)(t+)(t+)$ & $(-)(+t)(\mathrm{HH})(\mathrm{H})(\mathrm{H})(\mathrm{H})$ \\
\hline 23. & 11 A.M. & $18^{\circ} \mathrm{C}$ & tt) $(t+)(+t)(t+)$ & $(-)(+t)(H)(H+)(H)(H)$ \\
\hline $\mathbf{I}$ & 1 P.M. & $19^{\circ} \mathrm{C}$ & $(t+)$ & (H) $(H)(H)$ \\
\hline & & & & Ht) $(t+t)$ \\
\hline
\end{tabular}


As shown in Table II, all 40 samples of milk did not show a change in intensity of Arakawa's reaction even if the reaction was performed three hours later than the time of obtaining. This result coincides with that shown in Table I. If performed five hours later than the time of obtaining, the reaction showed a change in 21 samples, thought it was rather slight in degree. When performed 8 hours later than the time of obtaining, three samples showed a change, while the remaining 16 samples did not show any change even when tested at the eighth hour.

The result of all the 40 milk specimens may be summarised as follows :-

A change of the reaction in 3 hours

A change of the reaction in 5 hours

A change of the reaction in 8 hours

$0 \%$

No change of the reaction in the whole 8 hours $40 \%$

\section{2nd Series of Experiment.}

Milk samples were kept in an ice box. The result will be seen in the following Table III (Cf. Table III).

TABLE III.

Change of the same samples of the human milk from a lactants, kept in an ice box, as shown by means of Araliawa's reaction.

\begin{tabular}{|c|c|c|c|c|}
\hline \multirow{2}{*}{ Date } & \multirow{2}{*}{ Time } & \multirow{2}{*}{$\begin{array}{c}\text { Room } \\
\text { tempera- } \\
\text { ture }\end{array}$} & \multicolumn{2}{|c|}{ Arakawa's reaction } \\
\hline & & & $\begin{array}{c}\text { Right } \\
\left(0^{\prime}\right)\left(1^{\prime}\right)\left(2^{\prime}\right)\left(3^{\prime}\right)\left(4^{\prime}\right)\left(5^{\prime}\right)\end{array}$ & $\begin{array}{c}\text { Left } \\
\left(0^{\prime}\right)\left(1^{\prime}\right)\left(2^{\prime}\right)\left(3^{\prime}\right)\left(4^{\prime}\right)\left(5^{\prime}\right)\end{array}$ \\
\hline \multirow{4}{*}{$\begin{array}{l}17 . \\
I\end{array}$} & & $11^{\circ} \mathrm{C}$ & $(-)(t)(t+)(H)(H)(H)$ & )$(t t)(t t)(t+)(t t)$ \\
\hline & $11 \mathrm{~A}$. & 104 & $(-)(+)(++)(H+1)(H)(H)$ & $(t+)(t+)(t+)$ \\
\hline & & $\begin{array}{l}19^{\circ} \mathrm{C} \\
19^{\circ} \mathrm{C}\end{array}$ & $(-)(+)(++)(W)(H+)(H)$ & $(-)(+)(+t)(+t)(+t)(+t)$ \\
\hline & $\begin{array}{l}4 \text { P.M. } \\
8 \text { A.M. }\end{array}$ & $\begin{array}{l}19^{\circ} \mathrm{C} \\
16^{\circ} \mathrm{C}\end{array}$ & $(-)(+)(+t)(H)(H+)(H)$ & $(-)(+)(t+)(t+)(+t)(+t)$ \\
\hline \multirow{3}{*}{20} & 11 A.M. & $17^{\circ} \mathrm{C}$ & $(-\{(+)(t+)(+t)(+H)(H)$ & $(-)(+)(+t)(H)(H+)(H)$ \\
\hline & I P.M. & $18^{\circ} \mathrm{C}$ & $(-)(+)(+t)(+t)(H)(H)$ & (-) $(+)(+t)(H)(+H)$ W \\
\hline & $\begin{array}{l}4 \mathrm{P} \\
8 \mathrm{~A}\end{array}$ & $\begin{array}{l}17^{\circ} \mathrm{C} \\
17^{\circ} \mathrm{C}\end{array}$ & $(-)(+)(+t)(++)(+H)(H)$ & $(-)(+)(++)(++1)(+H)(H+)$ \\
\hline \multirow{3}{*}{$\stackrel{22 .}{I}$} & 11 A.M. & $19^{\circ} \mathrm{C}$ & $(-)(+)(+t)(H+)(H)(H)$ & $(-)(+)(t+)(t+)(t+)(t+)$ \\
\hline & $1 \mathrm{P}$ & $19^{\circ} \mathrm{C}$ & $(-)(+)(+t)(H)(H)(H)$ & $(-)(t)(t t)(t+)(t+)(t+1$ \\
\hline & & $\begin{array}{l}19^{\circ} \mathrm{C} \\
17^{\circ} \mathrm{C}\end{array}$ & $(-)(+)(+t)(+4)(+4)(+1)$ & $(-)(+)(+t)(t+)(t+)(t+)$ \\
\hline \multirow{3}{*}{$\underset{\mathrm{I}}{23 .}$} & $11 \mathrm{~A} . \mathrm{M}$. & $18^{\circ} \mathrm{C}$ & $(-)(+)(+t)(+t)(+t)(+t)$ & $\begin{array}{l}(-)(+t)((H)(H)(H)(H) \\
(-)(t+)(H+)(H+)(H+)(H+)\end{array}$ \\
\hline & $\begin{array}{l}1 \\
4 P .1\end{array}$ & $19^{\circ} \mathrm{C}$ & $(-)(+)(++)(++)(++)(++)$ & $(-)(++)(H)(H)(H)(H)$ \\
\hline & & & & \\
\hline
\end{tabular}




\begin{tabular}{|c|c|c|c|c|}
\hline \multirow[b]{2}{*}{ Date } & \multirow[b]{2}{*}{ Time } & \multirow{2}{*}{$\begin{array}{c}\text { Room } \\
\text { tempera- } \\
\text { ture }\end{array}$} & \multicolumn{2}{|c|}{ Arakawa's reaction } \\
\hline & & & $\begin{array}{l}\text { Right } \\
\left(0^{\prime}\right)\left(1^{\prime}\right)\left(2^{\prime}\right)\left(3^{\prime}\right)\left(4^{\prime}\right)\left(5^{\prime}\right)\end{array}$ & $\left(0^{\prime}\right)\left(1^{\prime}\right)\left(2^{\prime}\right)\left(3^{\prime}\right)\left(4^{\prime}\right)\left(5^{\prime}\right)$ \\
\hline & & & $(t+)(H t)(H t)(H)$ & $(-)(t)(t+)(H)(H+)(H)$ \\
\hline 27. & & & H) (HH) (HH) & $(-)(+)(t+)$ \\
\hline I & & & $(-)(+)(+t)(H)(H)(H)$ & $(-)(+)(t+)(H)(+H)(+H)$ \\
\hline & & & $(-)(+)(+t)(H)(+H)(+H)$ & $(-)(+)(++)(+H)(+H)(+H)$ \\
\hline & & & $(-)(t)(t)(t+)(t+)(t+)$ & $( \pm)(t+)(t+t)(H)(H)(H)$ \\
\hline 31. & 11 & & $(-)(t)(t)(t+)(t+)(t+)$ & $( \pm)(\mathrm{H})(\mathrm{H})(\mathrm{HH})(\mathrm{HH})(\mathrm{H})$ \\
\hline I & & & $(-)(+)(+)(t+)(t+)(t+)$ & $( \pm)(t+)(H)(H+)(H+)(H)$ \\
\hline & & & $(-)(+)(+)(t+)(t+)(t+)$ & $( \pm)(+t)(H)(H)(H)(H)$ \\
\hline & & & $(-)(+)(t+)(t+)(H+)(H)$ & $( \pm)(t+)(t+)(H)(H)(H)$ \\
\hline 7. & 11 & & $(-)(+)(t+)(t+)(H+)(+H)$ & $( \pm)(t+)(+t)(H)(H)(H)$ \\
\hline II & & & $(-)(+)(+t)(++)(H)(H)$ & $( \pm)(++)(++)(H)(H)(H)$ \\
\hline & & & $(-)(+)(t+)(t+)(H+)(H)$ & $( \pm)(t+)(+t)(H+)(H+)(H+)$ \\
\hline & & & $(-)(t+)(H)(H+H)(H)(H)$ & $( \pm)(+)(t+)(t+)(t+)(t+)$ \\
\hline 11. & & & $(-)(+t)(H+)(+H)(H)(H)$ & $( \pm)(t)(t+)(t+)(t+)(t+)$ \\
\hline II & & & $(-)(+t)(H+(H)(H)(H)$ & $( \pm)(+)(+t)(t+)(+t)(t+)$ \\
\hline & & & $(-)(t+)(H+)(H+)(H)(H)$ & $( \pm)(+)(++)(+t)(+t)(+t)$ \\
\hline & & & & $(-)(+t)(+t)(+t)(+H)(t$ \\
\hline $1 \mathrm{fi}$. & 112 & & $(+)(+)(t)(t)(+)$ & $(-)(t+)(t+)(+t)(H+)(H)$ \\
\hline II & & & $(-)(+)(+)(+)(+)(+)$ & $(-)(t+)(+t)(+t)(H)(H)$ \\
\hline & & & $+)(+)(+)(+)$ & $(-)(+t)(+t)(+t)(H)(H)$ \\
\hline & & & $(-)( \pm)(+)(+t)(+t)(H+)$ & $(-)( \pm)(+)(+)(+)(++)$ \\
\hline 19 & $11 \mathrm{~A}$ & $20^{\circ} \mathrm{C}$ & $( \pm)(+)(+t)(+t)(H)$ & $(-)( \pm)(+)(+)(+)(t+)$ \\
\hline & $\begin{array}{l}1 \text { P.M. } \\
4 \text { P.JI. }\end{array}$ & $\begin{array}{l}20^{\circ} \mathrm{C} \\
23^{\circ} \mathrm{C}\end{array}$ & 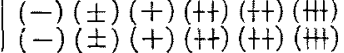 & $\mid \begin{array}{l}(-)( \pm)(+)(t)(t)(t+) \\
(-)( \pm)(t)(t)(t)(t+)\end{array}$ \\
\hline
\end{tabular}

As shown in Table III, all the 20 samples did not show any change of Arakawa's reaction in intensity, even if the reaction was performed eight hours later than the time of obtaining.

\section{Summary.}

The above experiment concerns the milk from only one lactant with a healthy growing infant. The experiment should be extented to more lactants, I admit, but it is very difficult to repeat such a systemmatic experiment with more lactants. From my experiment the following can be stated :-

1. A sample of fresh human milk with such an intensity as $(+t)^{*}$ $1^{\prime},(\mathrm{H})^{* *} 1^{\prime}$ or $(\mathrm{Ht})^{* * *} 2^{\prime}$ of A rakawa's reaction will keep the same or almost the same intensity as long as 8 hours, even if left to itself in summer time. Samples of human milk with weaker Arakawa's

* (tt) 1'.....A A ra k a a's reaction positive in the grade $(t t)$ in one minute.

** (HI) 1 .......A raka wa's reaction positive in the grade $(H+$ in one minute.

*** $(t+) 2^{\prime} \ldots .$. A r a k w $a^{\prime}$ 's reaction positive in the grade $(t+)$ in two minutes. 
reactions will begin to show a change of the intensity of reaction from the 5th hour on.

2. If a sample of fresh human milk is kept in an ice box, it will show the same intensity of Arakawa's reaction, even if tested as late as 8 hours after obtaining.

\section{Conclusions.}

Fresh human milk will keep the same intensity of Arakawa's reaction as at the time of milk obtaining, as long as 3 hours if left at room temperature even in summer time, and as long as 8 hours if kept in an ice box. 